\title{
Using 3D virtual surfaces to investigate molluscan shell shape
}

\author{
Massimiliano SCALici ${ }^{1}$, a Lorenzo TRAVERSETTI $^{1}$, Federica SPANI ${ }^{1}$, Raffaella BRAVI ${ }^{1}$, \\ Valentina MALAFOGLIA $^{1,2}$, Tiziana Persichini ${ }^{1}$ and Marco ColasANTI ${ }^{1}$ \\ ${ }^{1}$ Department of Science, University Roma Tre, viale Guglielmo Marconi 446, 00146 Rome, Italy \\ ${ }^{2}$ Institute for Research on Pain, ISAL-Foundation, Via San Salvador 204, 47922 Torre Pedrera, Rimini, Italy
}

Received 28 January 2016; Accepted 6 June 2016

\begin{abstract}
Noninvasive methods in shell shape variation may help to understand evolution, ecology, stress and role of molluscan in aquatic ecosystems. Imaging analysis is a suitable diagnostic tool in morphological studies to (1) evaluate the health status of investigated animals, and (2) monitor sea coastal habitats. We introduce the feasibility of the cone-beam computed tomography as an optimal technique for 3D surface scanning to obtain virtual valve surfaces of Mytilus galloprovincialis, and analyze them exploiting the geometric morphometric facilities. Statistical output revealed morphological difference between mussels coming from different extensive rearing systems highlighting how the entire valve surface contributed to discriminate between groups when we compared 2- and 3D analyses. Many factors drive the morphological differences observed in the valve shape variation between the two sites, such as geographical genetic differentiation, natural environmental effects and culture conditions. The simplicity of the proposed methodology avoids damage and handling of individuals, makes this approach useful for morphological data collection, and helps to detect detrimental agents for sea ecosystems by using molluscans.
\end{abstract}

Keywords: Mytilus galloprovincialis / cone-beam computed tomography / imaging / geometric morphometrics

\section{Introduction}

Investigation of bivalves using new noninvasive methods may contribute to better understanding of their ecology, evolution, and roles in aquatic ecosystems, overall as indicators of detrimental perturbations (Nuñez et al. 2012; Tosti and Gallo 2012). In this context we emphasized the use of morphological deformities as a tool to detect the exposition of an organism to chemicals, since the morphological abnormalities seem to increase with the disturbance degree (Le Cadre and Debenay 2006; Scalici et al. 2015).

Among the diverse methods, radiological techniques have shown promise in monitoring and morphological fields, as demonstrated by their recent application to living organisms (e.g. Bock et al. 2001, 2002; Jasanoff and Sun 2002; Mark et al. 2002; Wecker et al. 2002; Toussaint et al. 2005; Pouvreau et al. 2006; Golding and Jones 2007; Marxen et al. 2008), and fossils as well (e.g. Abel et al. 2012; Rahman et al. 2012; Adams et al. 2015).

Here, we introduce the feasibility and potential of 3D data acquisition and virtual reconstructions as an optimal technique for three-dimensional surfaces (such as those of bivalve shells) by using the cone-beam computed tomography (CBCT; for details see Scarfe and Farman 2008).

\footnotetext{
a Corresponding author:

massimiliano.scalici@uniroma3.it
}

In this work, we displayed, managed and analysed CBCT data from the mussel Mytilus galloprovincialis (Lamarck 1819) since it may act as a suitable tool to detect the environmental health in harvested ecosystems because of its intertidal habitat and close association with sediments (Bryan and Gibbs 1991). Indeed it is actually used as a biological model within the project "Systems Biology" (funded by Ministry of Education, University and Research, MIUR-PRIN, Italy), which is focused on environmental health status evaluation and potential recovery strategies for fisheries and/or aquaculture systems (Fasulo et al. 2015).

\section{Material and methods}

Briefly, our CBCT unit consists of a rotating gantry having (1) the fulcrum fixed within the center of the region of interest (i.e. field of view, FoV), (2) an X-ray scintillator layer (the X-ray source) at one extreme that directs a divergent coneshaped beam of ionizing radiation through the FoV, and (3) a detector based on a large-area solid-state sensor panel at the opposite extreme. From 150 to 600 sequential 2D planar projection images of the FoV may be acquired (at certain defined degree intervals) during a complete rotation. Once all the complete frame series has been acquired, the volume rendering allows the 3D surface to be displayed through integration and condensation of adjacent 2D images (virtual slices) into a 

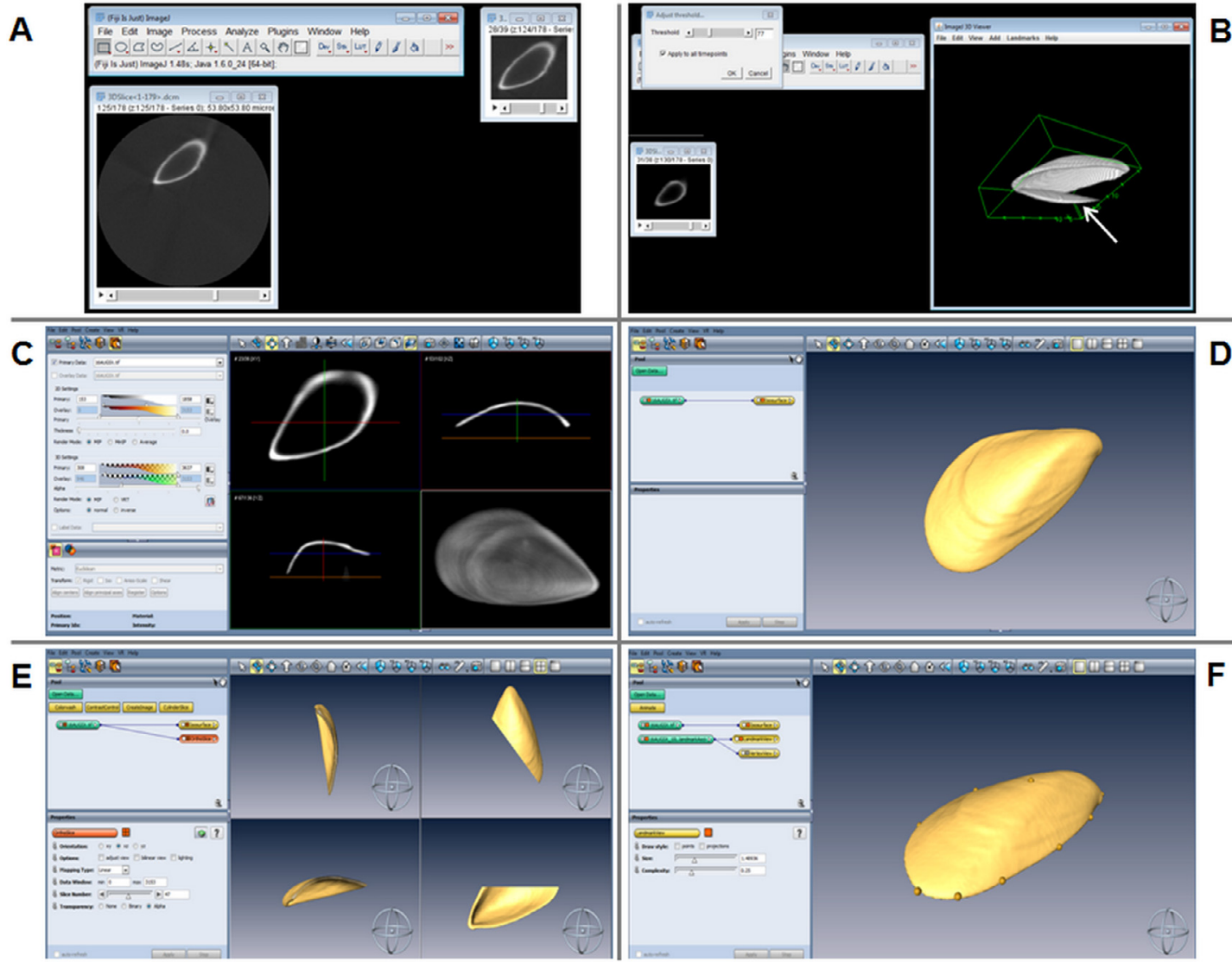

Fig. 1. Visualisation of vales of Mytilus galloprovincialis using Fiji: slice visualization and image field selection (A) and virtual image reconstruction (B); extraneous objects (such as the support, white arrow) may be deleted with other software (the green parallelepiped represents the new field of view, after removal of empty slices). Other visualization using Amira routines: three orthogonal section plans (C) of the 3D virtual mussel image (D), 3D cross-section (E) and example of 3D landmark fixation on the right valve external surface (F; for the exact position of all landmarks see Figs. 2A and 2B).

single object. The final data visualization may be either performed by the work station associated with the CBCT unit, or otherwise reconstructed using other software, depending on the visual interpretation and statistical analyses required.

The CBCT data (uploaded as DICOM images) of $M$. galloprovincialis were processed using the image-processing package Fiji (Schindelin et al. 2012) and the Amira ${ }^{\circledR} 5.2$ software package (Stalling et al. 2005).

In particular, the CBCT data were processed using first the image-processing package Fiji to perform a series of manipulations on each single $2 \mathrm{D}$ projection. The original volumetric data were first imported in Fiji (Fig. 1A) to reduce the FoV (green parallelepiped in Fig. 1B) by deleting all the empty exposures from the entire $2 \mathrm{D}$ projection series. In this way, we preserved only $2 \mathrm{D}$ projections containing image information on valves. It is also possible to eliminate artifacts or undesired objects from each 2D planar projection, and to save the new series of $2 \mathrm{D}$ projection data without noise that would produce a clean 3D-surface mesh. Then the new series of $2 \mathrm{D}$ projections can be uploaded into Amira, where each single slice can be visualized in the three main planes (Fig. 1C) and the investigated object displayed as a whole $3 \mathrm{D}$ reconstruction (Fig. 1D) or in cross sections (Fig. 1E) by applying the Isosurface module. The latter module computes a surface within a three-dimensional scalar field with regular Cartesian coordinates, and give the chance to set the threshold to determine the value used for the surface computation. Finally, the extracted surface may be saved in .ply format and used for following analyses. All this procedure guarantees a better volume rendering, since original 2D projections with noises may disturb the volume rendering by Amira, within which the ExtractSurface module allow to further complete the imaging purification process.

Since Amira provides an efficient platform to associate the 3D image handling with recent morphological techniques (Fig. 1F) (see also Monnet et al. 2009), we utilized the geometric morphometric (GM) method. GM overcomes the limitations of the traditional morphometrics by preserving the geometric properties of the studied objects (Adams et al. 2004). The $x$-, $y$ - and $z$-Cartesian coordinates of homologous landmarks (homologous points identified unambiguously from specimen to specimen) are used to describe the relative position of a part of the investigated object relative to another one (Zelditch et al. 2004). Our approach was to fix 3D landmarks in Amira (Fig. 1F) by using the Landmarks editor routine (Create $\rightarrow$ Data $\rightarrow$ Landmarks) that allowed to display a landmark set as small spheres. Then we employed the semilandmark procedure of Serb et al. (2011), in which shape 


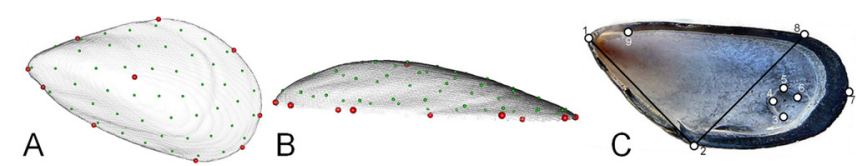

Fig. 2. Position of 3D landmarks (large spots) and semilandmarks (small spots) on (A) dorsal and (B) right-lateral views of Mytilus galloprovincialis left valve; (C) fixation of 2D landmarks on the left valve internal surface. The 2D landmark map: (1) umbo, (2) ligament, (3) posterior adductor 1, (4) posterior adductor 2, (5) posterior adductor 3, (6) posterior adductor 4, (7) posterior border, (8) projection from landmark 2, (9) anterior adductor.

information from landmarks as well as points along curves and points on the surface are included in the same analysis. Semilandmarks are nonhomologous points depending on landmarks (see Adams et al. 2004). We used 60 3D landmarks and semilandmarks (Figs. 2A and 2B) to quantify shell surface shape of 60 Mytilus right valves from 4.5-5.5 and 2.2-3.1 cm in length and width, respectively, and collected from two different aquaculture sites: Goro (Veneto, NE Italy; $n=29$ ) and Olbia (Sardinia, W Italy; $n=33$ ). Since no significant differences in size were found between the investigated sites in either length or width (Student's $t$ tests) we focussed only on shell shape variation. According to the protocol of Serb et al. (2011), we first fixed nine landmarks on the valve external edge and one on the point of highest elevation, and then produced a web of 50 semilandmarks across each single valve. The obtained point pattern was then used as template to be warped on the other valves by exploiting the fixed landmarks as points of correspondence. The semilandmark fixing procedure was run in $\mathrm{R}$ 3.2.4 (R Development Core Team 2009) using routines written by the authors. Once we obtained the point patterns for the studied valves, all the configurations were aligned by generalized Procrustes superimposition (GPS). This eliminates information on orientation and rotation (Rohlf and Slice 1990) and standardizes each valve to a unit centroid size (i.e. the square root of the summed squared Euclidean distances from each landmark to the specimen centroid that provides an estimate of the size of the studied structure; Zelditch et al. 2004). During this procedure, semilandmarks were permitted to slide to minimize Procrustes distance between specimens (Gunz et al. 2005; Perez et al. 2006). After the GPS procedure, we used the new set of shape coordinates as variables in multivariate statistical analyses (Mitteroecker and Bookstein 2008).

To show the efficacy of this 3D protocol used with the image capture tool, we compared three different GM approaches, using (1) the 2D landmarks fixed on planar images according to Valladares et al. (2010) (Fig. 2C), (2) only the 3D landmarks fixed in Amira (Fig. 1F), and (3) 3D landmarks plus semilandmarks collected as described. Before starting the statistical analyses, we digitized twice all 2D and 3D landmarks in all studied valves in order to estimate error due to point fixation, by Procrustes analysis of variance (ANOVA) (see Klingenberg 2011).

All descriptive (landmarks and semilandmark configuration inclusive) and statistical analyses were executed in R.

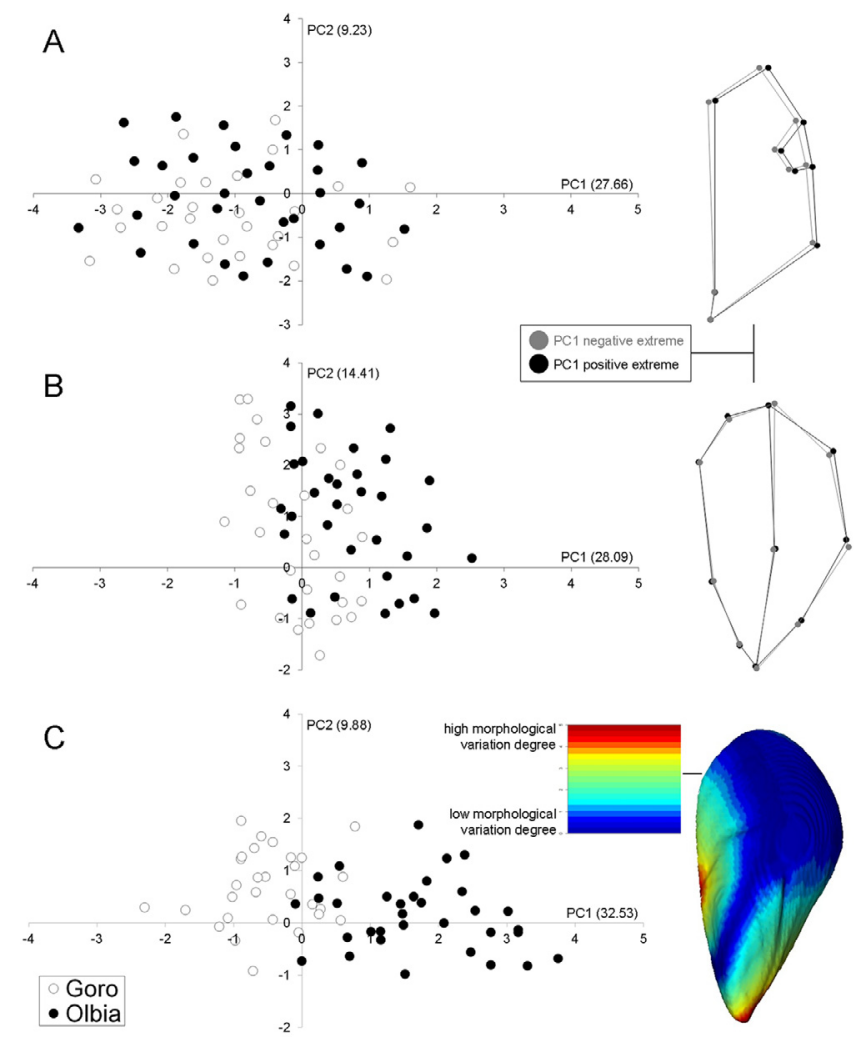

Fig. 3. Three scatter plots obtained by using the two first principal components (PCs) showing the pairwise comparisons of right valves of Mytilus galloprovincialis from the two aquaculture sites of Goro and Olbia. The graphical outputs resulted from the analyses of $2 \mathrm{D}$ landmarks (A), 3D landmarks only (B) and 3D landmarks plus semilandmarks (C). As for both A and B, the extreme configurations (negative in gray, and positive in black) for the main principal component (PC1) are shown on the right; as for $\mathrm{C}$, the associated configuration on the right showed the most variable shell portions from negative to positive extreme.

\section{Results and discussion}

The approach proposed in this study produces a relatively detailed, accurate and realistic representation of the bivalve shells, used in the following analyses.

The ANOVA showed that measurement error was negligible for the landmark digitalization on valves from the two sites in all three approaches $(P<0.001)$. Comparison of the three methods using the selected landmarks was therefore justified.

We first performed exploratory ordination analysis of the principal components to visualize the patterns of variation within each sample. The visual outputs for the three approaches were the three scatter plots reported in Figure 3, showing the first two principal components that explained $36.89,42.50$ and $42.41 \%$ of the variance for approaches (1), (2) and (3), respectively; each of the remaining components of the multivariate analyses performed on the three approaches explained less than 5\%. The Mahalanobis distances (Md) were calculated (with 10000 permutation runs) on the shape matrices for each of the three approaches, to evaluate the statistical 
significance of the differences between the samples from the two sites. At a confidence level $\alpha$ of 0.05 , Md revealed no differences between the sites using (1) the 2D landmark fixation technique $(M d=1.08, P=0.219)$, while significant differences emerged for both (2) 3D landmarks only $(M d=1.73$, $P=0.039)$, and (3) 3D landmarks + semilandmarks ( $M d=$ 3.62, $P=0.019)$.

Although bivalves are an excellent group for the application of GM because of their hard shells (Rufino et al. 2006), some problems may emerge in the image analyses. Initially, GM studies on bivalves used contour-based methods applied to the external edge (e.g. Ferson et al. 1985; Innes and Bates 1999; Scholz and Hartman 2007). More recently, some studies have employed a combination of landmarks, contours and sliding semilandmarks (Perez et al. 2006). These techniques have been successfully applied to distinguish between similar species of bivalves (Rufino et al. 2006; Costa et al. 2008), between wild and cultured stocks (Valladares et al. 2010), fossil and modern taxa (Aguirre et al. 2006), different wild populations (Rufino et al. 2012), and juveniles and adults in ontogenetic investigations (Márquez et al. 2010). Nevertheless, it has to be recognized that 2D analyses do not make use of all the morphological information in 3D structures, such as shells. Difficulties arise analysing the external shell shape of bivalves because there are generally few points exploitable as landmarks, or structures with clear homology. Indeed, the shell geometry is difficult to capture by conventional descriptive approaches, which may poorly capture the subtle differences, limiting their relevance as happens in the $2 \mathrm{D}$ analysis. It is clear in the scatter plots that while $2 \mathrm{D}$ analysis did not provide a separation of the two sites, their overlap progressively decreased when we used the 3D approaches, particularly the method of Serb et al. (2011) in which the entire valve surface contributed to the analysis. The same differences did not emerge using a planar picture and emerged only partially with a 3D image in which only one point is fixed on the point of highest elevation to take account of the volume of the object. This confirms the relevance in using the three-dimensional geometry as a tool of great potential for the study of the anatomy of marine Mollusca. However, to date, morphological studies of molluscs by using the computed tomography approach integrated to geometric morphometric analyses remain rare, and under exploited (see Monnet et al. 2009).

Our approach provides a basis for the quantitative analysis of intraspecific morphological variation patterns, overall in taxa considered good environmental monitors. Indeed, the main aim was to propose the morphological alterations in the valve shell shape of $M$. galloprovincialis to detect how sea habitat features may affect both population and ecosystem health.

Many factors may drive the morphological differences observed in the valve shape variation between the two aquaculture sites, such as geographical genetic differentiation, natural environmental effects, and culture conditions. Although our sampling design included sites which differ in environmental features, the influence of multiple stressors cannot be neglected. Anyway, their study is beyond the scope of the present methodological demonstration. Although our study could not differentiate among the real sources of variation responsible for the observed patterns, these efforts support the value in using morphological markers as a useful early warning systems.

Here, we have shown an example of the efficacy of the application of CBCT associated with the GM technique on bivalve shells. The simplicity of the proposed methodology, based on noninvasive procedures (avoiding potential damage and handling of individuals), makes this approach a useful one for quantifying the mollusc shell geometry of specimens from different harvested habitats. It allows to observe the whole surface without destroying the sample, which is helpful when (1) working with type specimens of museums (used to define species), (2) when we have one or very few specimens, and/or (3) specimens have to be used for further analyses (e.g., ecotoxicological analyses on both shell and soft tissue).

The main emerging output is the evidence in using a potential fishery resource as a prognostic indicator of the habitat condition, and to propose a new tool for the exploration of scenarios and strategies for the conservation of aquatic biodiversity, aimed at ensuring sustainable and a high quality exploitation of aquatic living resources.

Acknowledgements. We are indebted to Dr. David G. Reid to improve the manuscript with their helpful comments and suggestions, and to Dr. Marco Moscato for his kindness to allows the use of the CBCT system. This investigation was supported by MIUR-PRIN grant to MC (2010ARBLT7_001/008).

\section{References}

Abel R.L., Laurini C.R., Richter M., 2012, A palaeobiologist's guide to "virtual" micro-CT preparation. Palaeontol. Electr. 15, 6T, 17p.

Adams D.C., Rohlf F.J., Slice D.E., 2004, Geometric morphometrics: ten years of progress following the "revolution". Ital. J. Zool. 71, $5-16$.

Adams J.W., Olah A., McCurry M.R., Potze S., 2015, Surface Model and Tomographic Archive of Fossil Primate and Other Mammal Holotype and Paratype Specimens of the Ditsong National Museum of Natural History, Pretoria, South Africa. PLoS ONE 10, e0139800.

Aguirre M.L., Perez S.I., Sirch Y.N., 2006, Morphological variability of Brachidontes Swainson (Bivalvia, Mytilidae) in the marine Quaternary of Argentina (SW Atlantic). Palaeogeogr. Palaeocl. Palaeoecol. 239, 100-125.

Bock C., Frederich M., Wittig R., Pörtner H.O., 2001, Simultaneous observations of haemolymph flow and ventilation in marine spider crabs at different temperatures: a flow weighted MRI study. Mag. Res. Imag. 19, 113-1124.

Bock C., Sartoris F.J., Pörtner H.O., 2002, In vivo MR spectroscopy and MR imaging on non-anaesthetized marine fish: techniques and first results. Mag. Res. Imag. 20, 165-172.

Bryan G.W., Gibbs P.E., 1991, Impact of low concentrations of tributyltin (TBT) on marine organisms: a review. In: Newman M.C., Mc Intosh A.W. (Eds.), Metal Ecotoxicology: Concepts and Applications, Chelsea, Michigan, Lewis Publishers, pp. 323361.

Costa C., Aguzzi J., Menesatti F., Antonucci F., Rimatori V., Mattoccia M., 2008, Shape analysis of different populations of clams in relation to their geographical structure. J. Zool. 276, 7181 . 
Fasulo S., Guerriero G., Cappello S., Colasanti M., Schettino T., Leonzio C., Mancini G., Gornati R., 2015, The "SYSTEMS BIOLOGY" in the study of xenobiotic effects on marine organisms for evaluation of the environmental health status: biotechnological applications for potential recovery strategies. Rev. Env. Sci. Bio.-Tech. 14, 339-345.

Ferson S.F., Rohlf F.J., Koehn R.K., 1985, Measuring shape variation of two-dimensional outlines. Syst. Zool. 34, 59-68.

Golding R.E., Jones A.S., 2007, Micro-CT as a novel technique for 3D reconstruction of molluscan anatomy", Molluscan Res. 27, 123-128.

Gunz P., Mitteroecker P., Bookstein F.L., 2005, Semilandmarks in three dimensions. In: Slice D.E. (Ed.), Modern morphometrics in physical anthropology, New York, Kluwer Academic/Plenum Publishers, pp. 73-98.

Innes D.J., Bates J.A., 1999, Morphological variation of Mytilus edulis and Mytilus trossulus in eastern Newfoundland. Mar. Biol. 133, 691-399.

Jasanoff A., Sun P., 2002, In vivo magnetic resonance microscopy of brain structure in unanesthetized flies. J. Mag. Resonan. 158, $79-85$.

Klingenberg C.P., 2011, MorphoJ: An integrated software package for geometric morphometrics. Mol. Ecol. Res. 11, 353-357.

Le Cadre, V., Debenay, J.-P., 2006. Morphological and cytological responses of Ammonia (foraminifera) to copper contamination: Implication for the use of foraminifera as bioindicators of pollution. Environ. Pollution 143, 304-317.

Mark F.C., Bock C., Pörtner H.O., 2002, Oxygen limited thermal tolerance in Antarctic fish investigated by magnetic resonance imaging (MRI) and spectroscopy ( $\left.{ }^{31} \mathrm{P}-\mathrm{MRS}\right)$. Am. J. Physiol. Regul. Integr. Comp. Physiol. 283, 1254-1262.

Márquez F., Amoroso R., Sainz M.F.G., Van der Molen S., 2010, Shell morphology changes in the scallop Aequipecten tehuelchus during its life span: a geometric morphometric approach. Aquat. Biol. 11, 149-155.

Marxen J.C., Prymak O., Beckmann F., Neues F., Epple M., 2008, Embryonic shell formation in the snail Biomphalaria glabrata: a comparison between scanning electron microscopy (SEM) and synchrotron radiation micro computer tomography (SR $\mu \mathrm{CT})$. J. Molluscan Stud. 74, 19-25.

Mitteroecker P., Bookstein F., 2008, The evolutionary role of modularity and integration in the hominoid cranium. Evolution 62, 943-958.

Monnet C., Zollikofer C., Bucher H., Goudemand N., 2009, Three-dimensional Morphometric Ontogeny of Mollusc Shells by Micro-Computed Tomography and Geometric Analysis. Palaeontol. Electr. 12, 12A, 13p.

Nuñez J.D., Laitano M.V., Cledón M., 2012, An intertidal limpet species as a bioindicator: pollution effects reflected by shell characteristics. Ecol. Indic. 14, 178-183.

Perez S.I., Bernal V.,Gonzalez P.N., 2006, Differences between sliding semi-landmark methods in geometric morphometrics, with an application to human craniofacial and dental variation. J. Anat. 208, 769-784.

Pouvreau S., Rambeau M., Cochard J.C., Robert R., 2006, Investigation of marine bivalve morphology by in vivo MR imaging: first anatomical results of a promising technique. Aquaculture 259, 415-423.
R Development Core Team, 2009, R: a language and environment for statistical computing. Version 2.91. Available at http://cran.Rproject.org R Foundation for Statistical Computing, Vienna.

Rahman I.A., Adcock K., Garwood R.J., 2012, Virtual Fossils: a New Resource for Science Communication in Paleontology. Evo. Educ. Outreach 5, 635-641.

Rohlf F.J., Slice D.E., 1990, Extensions of the Procrustes method for the optimal superimposition of landmarks. Syst. Zool. 39, 40-59.

Rufino M.M., Gaspar M.B., Pereira A.M., Vasconcelos P., 2006, Use of shape to distinguish Chamelea gallina and Chamelea striatula (Bivalvia: Veneridae): linear and geometric morphometric methods. J. Morph. 267, 1433-1440.

Rufino M.M., Vasconcelos P., Pereira F., Fernández-Tajes J., Darriba S., Méndez J., Gaspar M.B., 2012, Geographical variation in shell shape of the pod razor shell Ensis siliqua (Bivalvia: Pharidae). Helgoland Mar. Res. 67, 49-58.

Scalici M., Traversetti L., Malafoglia V., Persichini T., Colasanti M., 2015, Concepts and tools for exploiting sessile bio-filters as early warning elements: introductory applications for marine ecosystem preservation. Inv. Surv. J. 12, 278-286.

Scarfe W.C., Farman A.G., 2008, What is cone-beam CT and how does it work? Dental Clin. North Am. 52, 07-730.

Schindelin J., Arganda-Carreras I., Frise E., Kaynig V., Longair M., Pietzsch T., Preibisch S., Rueden C., Saalfeld S., Schmid B., Tinevez J.Y., White D.J., Hartenstein V., Eliceiri K., Tomancak P., Cardona A., 2012, Fiji: an open-source platform for biologicalimage analysis. Nat. Meth. 9, 676-682.

Scholz H., Hartman J.H., 2007, Fourier analysis and the extinction of unionoid bivalves near the Cretaceous-Tertiary boundary of the Western Interior, USA: pattern, causes, and ecological significance. Palaeogeogr. Palaeocl. Palaeoecol. 255, 48-63.

Serb J.M., Alejandrino A., Otarola-Castillo E., Adams D.C., 2011, Morphological convergence of shell shape in distantly related scallop species (Mollusca: Pectinidae). Zool. J. Linn. Soc. 163, 571-584.

Stalling D., Westerhoff M., Hege H.C., 2005, Amira: a highly interactive system for visual data analysis. In: Hansen C.D., Johnson C.R., (Eds.), The Visualization Handbook, Amsterdam, Elsevier, pp 749-767.

Tosti E., Gallo A., 2012, Best biomarker and bioindicator for marine environmental pollution. J. Mar. Sci. Res. Dev. 2, 2.

Toussaint C., Fauconneau B., Medale F., Colleweta G., Akokad S., Haffraye P., Davenel A., 2005, Description of the heterogeneity of lipid distribution in the flesh of brown trout (Salmo trutta) by MR imaging. Aquaculture 243, 255-267.

Valladares A., Manríquez G., Suírez-Isla B.A., 2010, Shell shape variation in populations of Mytilus chilensis (Hupé 1854) from southern Chile: a geometric morphometric approach. Mar. Biol. 157, 2731-2738.

Wecker S., Hörnshemeyer T., Hoehn M., 2002, Investigation of insect morphology by MRI: Assessment of spatial and temporal resolution. Mag. Res. Imag. 20, 105-111.

Zelditch M.L., Swiderski D.L., Sheets H.D., Fink W.L., 2004, Geometric morphometrics for biologists: a primer. Amsterdam, Elsevier/Academic Press. 